\title{
Wei et al. on "Effect of ball milling and heat treatment process on MnBi powders magnetic properties JALCOM-D-15-09718"
}

Effect of ball milling and heat treatment process on MnBi powders magnetic properties

2 Wei Xie $^{1,2, \text { a }}$, Evgueni Polikarpov ${ }^{2}$, Jung-Pyung Choi ${ }^{2}$, Mark E Bowden ${ }^{3}$, Kewei Sun ${ }^{4}$, Jun Cui ${ }^{2,4}$

3 1. Longyan Redbud Innovation Research Institute, 1\# Dongxiao North Road, Xinluo District,

$4 \quad$ Longyan City, Fujian Province, P.R. China

5 2. Pacific Northwest National Laboratory, 902 Battelle Blvd., P.O. Box 999, Richland, WA 699352, USA

7 3. Environmental Molecular Sciences Laboratory, Pacific Northwest National Laboratory, 902

8 Battelle Blvd., P.O. Box 999, Richland, WA 99352, USA

9 4. Department of Materials Science and Engineering, Iowa State University, Ames, IA 50011, 10 USA

11 Corresponding author: Wei Xie, E-mail: xieweixw2000@yahoo.com, TEL.: +86 15280818515

\section{Abstract}

13 The metallic compound $\mathrm{MnBi}$ has high intrinsic coercivity with large positive temperature 14 coefficient. The coercivity of $\mathrm{MnBi}$ exceeds $12 \mathrm{kOe}$ and $26 \mathrm{kOe}$ at $300 \mathrm{~K}$ and $523 \mathrm{~K}$, 15 respectively. Hence $\mathrm{MnBi}$ is a good candidate for the hard phase in exchange coupled nanocomposite magnets. In order to maximize the loading of the soft phase, the size of the MnBi

17 particle has to be close to $500 \mathrm{~nm}$, the size of single magnetic domain. Low energy milling is the common method to reduce MnBi particle size. However, only $3-7$ micron size particle can be

19 achieved without significant decomposition. Here, we report our effort on preparing submicron

$20 \mathrm{MnBi}$ powders using traditional powder metallurgy methods. $\mathrm{Mn}_{55} \mathrm{Bi}_{45}$ magnetic powders were

21 prepared using arc melting method, followed by a series of thermal-mechanical treatment to 


\section{Wei et al. on "Effect of ball milling and heat treatment process on MnBi powders magnetic properties JALCOM-D-15-09718"}

improve purity, and finished with low energy ball milling at cryogenic temperature to achieve submicron particle size. The $\mathrm{Mn}_{55} \mathrm{Bi}_{45}$ powders were decomposed during ball milling process and recovered during 24 hours $290^{\circ} \mathrm{C}$ annealing process. With increasing ball-milling time, the saturation magnetization of $\mathrm{MnBi}$ decreases, while the coercivity increases. Annealing after ball milling recovers some of the magnetization, indicating the decomposition occurred during the ball-milling process can be reversed. The coercivity of $\mathrm{Mn}_{55} \mathrm{Bi}_{45}$ powders are also improved as a result of the heat treatment at $290{ }^{\circ} \mathrm{C}$ for 24 hours. The world record magnetization $71.2 \mathrm{emu} / \mathrm{g}$ measured applying a field of $23 \mathrm{kOe}$ has been achieved via low energy ball mill at room temperature.

Key words: low energy ball milling, low temperature phase, MnBi powders, rare earth free permanent magnet

\section{Introduction}

Rare-earth-free permanent magnets have attracted great attention due to the criticality of rare earth elements such as $\mathrm{Nd}$ and $\mathrm{Dy}$. MnBi permanent magnet as an alternative to rare-earth based permanent magnets has drawn attention due to its unique magnetic properties, especially the unusual positive temperature coefficient of coercivity $[1.45 \% / \mathrm{K}]$ in the temperature range $300-$ $530 \mathrm{~K}$ [1]. Its coercivity increases with increasing temperature, reaching $25 \mathrm{kOe}$ at $540 \mathrm{~K}$ [2-5] and up to $28.3 \mathrm{kOe}$ at $530 \mathrm{~K}$ as recently reported by Rao [6]. This relatively large coercivity of the $\mathrm{MnBi}$ in its low-temperature phase (LTP) is attributed to its high uniaxial magnetocrystalline anisotropy $\left(1.6 \times 10^{7} \mathrm{erg} \mathrm{cm}^{-3}\right.$ at $300 \mathrm{~K}$ and $2.3 \times 10^{7} \mathrm{erg} \mathrm{cm}^{-3}$ at $\left.420 \mathrm{~K}\right)$ [1]. However, $\mathrm{MnBi}$ has relatively low room temperature saturation magnetization $(75 \mathrm{emu} / \mathrm{g}$ measured with $23 \mathrm{kOe}$ applied field [7] or $81 \mathrm{emu} / \mathrm{g}$ measured applying a field of $90 \mathrm{kOe}$ [8]). Despite the large intrinsic 


\section{Wei et al. on "Effect of ball milling and heat treatment process on MnBi powders magnetic properties JALCOM-D-15-09718"}

coercivity, the theoretical maximum energy product of $\mathrm{MnBi}$ at room temperature is about 17 MGOe, much lower than that of NdFeB or SmCo.

One possible approach to improve MnBi's energy product is to exchange-couple it with a soft phase such as Co or FeCo [9-11]. This approach requires the size of the MnBi particles to be comparable to the size of its single magnetic domain $(\sim 500 \mathrm{~nm})[12,13]$. Obtaining submicron MnBi powder with small size distribution and high purity is critical for a successful implementation of this approach. The most popular method to prepare $\mathrm{MnBi}$ powder is low energy ball milling because high mechanical energy input may lead to decomposition. Ballmilling at low temperature can mitigate the decomposition issue, but not completely. $\mathrm{MnBi}$ particles with size in the range of $400-500 \mathrm{~nm}$ can be prepared using cryo-milling method, but the decomposition is still significant. The resulted powders had a magnetization $\sim 35 \mathrm{emu} / \mathrm{g}$ measured under $30 \mathrm{kOe}$ applied field at room temperature, indicating nearly half of the LTP phase has decomposed [14]. Yin [15] attributed the decomposition to the strains accumulated in the crystals of $\mathrm{MnBi}$ during ball milling process. $\mathrm{MnBi} / \mathrm{Bi}$ composite nanoparticles with size in the range 100-300 $\mathrm{nm}$ exhibiting anisotropic magnetic properties and high coercivity $16.3 \mathrm{kOe}$ were fabricated using the mechanochemical synthesis method [16], however, the powder magnetization only reached $22 \mathrm{emu} / \mathrm{g}$ measured with $30 \mathrm{kOe}$ applied field indicating large amount of impurity. Direct chemical synthesis of MnBi particles were carried out, but similar to the mechanochemical method, the resulted powder has low magnetization $(\sim 20 \mathrm{emu} / \mathrm{g})$ [17]. It appears that a new method is needed to fabricate MnBi powder with high purity (magnetization $>60 \mathrm{emu} / \mathrm{g}$ measured applying a field of $20 \mathrm{kOe}$ ) and with the desired $500 \mathrm{~nm}$ particle size. Here, 


\section{Wei et al. on "Effect of ball milling and heat treatment process on MnBi powders magnetic properties JALCOM-D-15-09718"}

we report our effort on fabrication of submicron MnBi particles using a combination of low temperature low energy ball milling and annealing.

\section{Experiment}

$\mathrm{Mn}_{55} \mathrm{Bi}_{45}$ powder was prepared using arc melting followed by grinding, annealing and low energy ball milling (LEBM). $\mathrm{Mn}_{55} \mathrm{Bi}_{45}$ alloys were prepared from high purity (> $99.95 \%$ ) elements (Aldrich and Alfa Aesar) by arc melting evacuated to $1 \times 10^{-5}$ Torr. The mixture of raw elements was pressed into a pellet using a uniaxial press before arc melting. The alloy was annealed in pure hydrogen atmosphere to obtain high-purity low temperature phase (LTP) MnBi. The obtained ingot was pulverized into powder using mortar and pestle. The powders were sieved using \#325 and \#400 mesh screens. The particles size range is $37-44 \mu \mathrm{m}$. The powders were heat treated under vacuum at $290^{\circ} \mathrm{C}$ for $48 \mathrm{~h}$ to increase LTP MnBi phase volume fraction. The MnBi powders were ball milled with LEBM either at room temperature or cryogenic environment (liquid nitrogen, $-70{ }^{\circ} \mathrm{C}$ ) respectively. 5 grams of $\mathrm{Mn}_{55} \mathrm{Bi}_{45}$ powders were loaded in the glove box in a hardened stainless steel vial with toluene at room temperature and hexane at $-70{ }^{\circ} \mathrm{C}$. The milling media were chrome steel balls from $4-12 \mathrm{~mm}$ in diameter. The ball to powders weight ratio was 20:1. The powders were milled for $3,8,24$ hours respectively with a milling speed of $60 \mathrm{rpm}$ and heat treated for at $290^{\circ} \mathrm{C}$ for 24 hours, then ball milled for half an hour with the same milling speed.

Powder diffraction data were collected with a Panalytical MPD Bragg-Brentano diffractometer using $\mathrm{Cu} \mathrm{K} \alpha$ radiation $(\lambda=1.5416 \AA$ ), a graphite post-diffraction monochromator, and variable divergence and anti-scatter slits (illuminated length - $10 \mathrm{~mm}$ ). The x-ray tube was operated at $50 \mathrm{kV}$ and $40 \mathrm{~mA}$ and patterns were collected between 20 and $100^{\circ} 2 \theta$, counting for $2 \mathrm{~s}$ at $0.04^{\circ}$ 


\section{Wei et al. on "Effect of ball milling and heat treatment process on MnBi powders magnetic properties JALCOM-D-15-09718"}

87

intervals. Phase proportions, cell parameters, and crystallite sizes were determined by wholepattern Rietveld fitting using the fundamental parameters approach implemented in Topas (v4.2, Bruker AXS) and crystal structures from the Inorganic Crystal Structure Database. For MnO where the diffraction peaks were weak and relatively broad, the lattice parameter was not refined and the crystallite size was also fixed at $50 \mathrm{~nm}$.

The magnetic properties were obtained using a vibrating sample magnetometer (VSM Model EZ9, Microsense) at room temperature with a maximum field of $23 \mathrm{kOe}$. Particle morphology and composition were investigated by scanning electron microscope (JSM-7001F, JEOL USA, Inc. Peabody, MA) with EDS capability (EDAX Li-drifted Si detector, AMETEK, Berwyn, PA). The focused ion beam technique was used to prepare samples for transmission electron microscopy (TEM), which was used to observe the microstructure of MnBi powders.

\section{Result and discussion}

Representative SEM morphologies of ball milled $\mathrm{Mn}_{55} \mathrm{Bi}_{45}$ for varied milling times are demonstrated in Fig. 1. The Figure shows that the particle size decreases with increased milling time. With an initial powder size before LEBM being $37-44 \mu \mathrm{m}$, the particle size is reduced to $1-4 \mu \mathrm{m}, 400 \mathrm{~nm}-2 \mu \mathrm{m}, 50 \mathrm{~nm}-1 \mu \mathrm{m}$ by 3,8 , and 24 hours of ball milling, respectively.

TEM microscopy and the corresponding selected area diffraction (SAD) patterns of the $\mathrm{Mn}_{55} \mathrm{Bi}_{45}$ powders ball milled for 3 hours and 24 hours are shown in Fig. 2. The SAD patterns of the $\mathrm{Mn}_{55} \mathrm{Bi}_{45}$ powders show presence of an amorphous phase in the ball-milled powders.

The X-Ray diffraction patterns of $\mathrm{Mn}_{55} \mathrm{Bi}_{45}$ powders ball milled for 8 hours, then heat-treated, and then ball milled for half hour are shown in Fig. 3. The peaks become broadened after ball 


\section{Wei et al. on "Effect of ball milling and heat treatment process on MnBi powders magnetic properties JALCOM-D-15-09718"}

milling indicating a reduction in grain size. The peaks get narrowed after heat treatment demonstrating a growth in grain size.

Table 1 shows X-Ray phase composition analysis of MnBi powders prepared by 8 hours of ball milling, followed by heat-treatment for 24 hours and then ball milling for half hour. The fractions of the magnetic $\mathrm{MnBi}$ phase are 77, 85.9, 81, 86.1 wt. \% for ball milled, milled and annealed, milled plus annealed and milled, and the feedstock (before ball milling) MnBi powders respectively. The fractions of $\mathrm{Bi}$ are 13.7, 5.2, 10.2, and 5.0 wt. $\%$ for ball milled, milled and annealed, milled plus annealed and milled, and feedstock MnBi powders respectively. The fraction of $\mathrm{MnBi}$ deceases and that of $\mathrm{Bi}$ increases after ball-milling indicating there is decomposition. Ball milling was followed by annealing which resulted in partial recovery of the MnBi phase. This observation is in agreement with previously published results [18]. The phase fractions of $\mathrm{Mn}$ and $\mathrm{MnO}$ remain fairly constant (the sum is 8.7 to $9.3 \mathrm{wt} \%$ ). The average crystallite sizes calculated from XRD patterns are 52, 115, 85 and $215 \mathrm{~nm}$ for ball milled, milled and annealed, milled plus annealed and milled, original $\mathrm{MnBi}$ powders respectively. The crystallite sizes were obtained from the peak widths (Scherrer broadening) in excess of that calculated from instrumental factors during the whole-pattern fitting. Note that the crystallite size is a different measurement than particle size; the former estimates the domain size which contains a more or less perfect crystal lattice while the latter is the physical dimension of individually separable grains of material. The crystallite size of $\mathrm{MnBi}$ and $\mathrm{Mn}$ decreases as a result of ball milling. The $\mathrm{Bi}$ fraction was small to begin with and decreases much less noticeably. The lattice parameters increase slightly indicating that the strain of MnBi phase goes up as well during ball milling process. The strains caused by ball milling process are difficult to 


\section{Wei et al. on "Effect of ball milling and heat treatment process on MnBi powders magnetic properties JALCOM-D-15-09718"}

130

131

132

133

134

135 136

137 138

139

140

141

142

143

144

145

146

147

148

149

150

151 accumulate in the crystals of $\mathrm{MnBi}$ and lead to the decomposition. Yin [15] also reported that after ball milling over 2 hours, Bi is precipitated from the LTP MnBi phase.

Magnetization curves of $\mathrm{Mn}_{55} \mathrm{Bi}_{45}$ powders prepared by three, eight and twenty four hours of ball milling are shown in Fig. 4. The saturation magnetization Ms is reduced with increasing milling time, which indicates decomposition during ball milling. On the other hand, the coercivity Hc is increased with increasing ball-milling time, in accordance with the $\mathrm{MnBi}$ particle size reduction. Coercivity reaches its maximum at eight hours of ball milling. There are kinks on the magnetization curves of $\mathrm{Mn}_{55} \mathrm{Bi}_{45}$ powders prepared by eight and twenty four hours ball milling (see Fig. 4).

The comparison of magnetization curves of $\mathrm{Mn}_{55} \mathrm{Bi}_{45}$ powders prepared for eight hours ball milling, then heat-treated, and then ball milled again for half hour is demonstrated in Fig. 5. There is a kink in magnetization curves of $\mathrm{Mn}_{55} \mathrm{Bi}_{45}$ powders after eight hours ball milling, possibly due to the presence of amorphous phase. After the 24 hours heat treatment at $290{ }^{\circ} \mathrm{C}$, the coercivity $\mathrm{Hc}$ is significantly improved. This indicates that the amorphous phase has been crystalized to recover some of the magnetic MnBi phase. However the magnetization Ms of $\mathrm{Mn}_{55} \mathrm{Bi}_{45}$ powders measured at $23 \mathrm{kOe}$ applied field is slightly decreased. This is attributed to the unsaturated magnetization of MnBi powder. Measurements at higher fields should help achieve saturation. The coercivity $\mathrm{Hc}$ of $\mathrm{Mn}_{55} \mathrm{Bi}_{45}$ is reduced for only half hour ball milling after $290{ }^{\circ} \mathrm{C}$ for $48 \mathrm{~h}$ heat treatment. The record of magnetization $71.2 \mathrm{emu} / \mathrm{g}$ is achieved via milling for eight hours, heat treated, and ball milled for extra half an hour process.

The comparison of morphology of ball milled $\mathrm{Mn}_{55} \mathrm{Bi}_{45}$ powders either at room temperature and cryogenic environment are demonstrated in Fig.6. Cryo ball milled $\mathrm{Mn}_{55} \mathrm{Bi}_{45}$ powders have less

[键入文字]

Page 7 


\section{Wei et al. on "Effect of ball milling and heat treatment process on MnBi powders magnetic properties JALCOM-D-15-09718"}

agglomeration. Magnetization curves of ball milled $\mathrm{Mn}_{55} \mathrm{Bi}_{45}$ powders at room temperature and cryogenic environment for 8 hours and 24 hours are shown in Fig. 7 and Fig. 8 respectively. The magnetization of ball milled $\mathrm{Mn}_{55} \mathrm{Bi}_{45}$ powders for 8 hours are $65.4 \mathrm{emu} / \mathrm{g}$ and $63.2 \mathrm{emu} / \mathrm{g}$ at cryogenic environment and room temperature respectively. The magnetization of ball milled $\mathrm{Mn}_{55} \mathrm{Bi}_{45}$ powders for 24 hours $55.5 \mathrm{emu} / \mathrm{g} 47.8 \mathrm{emu} / \mathrm{g}$ at cryogenic environment and room temperature respectively. The magnetization of ball milled $\mathrm{Mn}_{55} \mathrm{Bi}_{45}$ powders at cryogenic environment is higher than that at room temperature for both 8 hours and 24 hours ball milling. This indicates that the cryo ball milling retards decomposition. However this function is reduced after the ball milling time is above 8 hours. Both coercivity of ball milled $\mathrm{Mn}_{55} \mathrm{Bi}_{45}$ powders for 8 hours and 24 hours at cryogenic environment are lower than that at room temperature.

\section{Conclusion}

The $\mathrm{Mn}_{55} \mathrm{Bi}_{45}$ powders size is reduced dramatically from $44 \mu \mathrm{m}$ to $1-4 \mu \mathrm{m}, 400 \mathrm{~nm}-2 \mu \mathrm{m}$, $50 \mathrm{~nm}-1 \mu \mathrm{m}$ by $3,8,24$ hours ball milling respectively. The MnBi powders with grain size close to magnetic domain, i.e. $500 \mu \mathrm{m}$, can be produced via ball milling process and used as hard phase for exchange coupled nanocomposite magnets. An amorphous phase was found in 24 hours ball milled powders through TEM analysis. The XRD analysis demonstrates that the $\mathrm{Mn}_{55} \mathrm{Bi}_{45}$ powders were decomposed during ball milling process and recovered during 24 hours $290{ }^{\circ} \mathrm{C}$ annealing process. The saturation magnetization $\mathrm{Ms}$ is reduced with increasing ball milling time, which indicates that the decomposition is increased with increasing ball milling time. The coercivity of $\mathrm{Mn}_{55} \mathrm{Bi}_{45}$ powders are improved as a result of the heat treatment at $290{ }^{\circ} \mathrm{C}$ for 24 hours, indicating that the magnetic MnBi phase has been partially recovered. The 


\section{Wei et al. on "Effect of ball milling and heat treatment process on MnBi powders magnetic properties JALCOM-D-15-09718"}

world record magnetization $71.2 \mathrm{emu} / \mathrm{g}$ has been achieved via low energy ball mill at room

temperature.

\section{Acknowledgements}

This research was supported by the US Department of Energy (DOE) Advanced Research Projects Agency- Energy (ARPA-E) under Contract No. 11/CJ000/09/03. Ames Laboratory is operated for the US DOE by Iowa State University under contract \#DE-AC02-07CH11358. Special thanks to prof. Jin Bo Yang from Peking University and prof. Don Lee from Chinese Academy of Science for their kind discussion.

\section{References}

[1] X. Guo, X. Chen, Z. Altounian, J.O. Ström-Olsen, Magnetic properties of MnBi prepared by rapid solidification, Phys. Rev. B 46 (1992) 14578-14582.

[2] Y.B. Yang, X.G. Chen, S. Guo, A.R. Yan, Q.Z. Huang, M.M. Wu, D.F. Chen, Y.C. Yang, J.B. Yang, Temperature dependences of structure and coercivity for melt-spun MnBi compound, J. Magn. Magn. Mater., 330 (2013) 106-110.

[3] J.B. Yang, W.B. Yelon, W.J. James, Q. Cai, M. Kornecki, S. Roy, N. Ali, P. L'Heritier, Crystal structure, magnetic properties and electronic structure of the $\mathrm{MnBi}$ intermetallic compound, J. Phys.: Condens. Matter, 14 (2002) 6509-6519.

[4] J.B. Yang, Y.B. Yang, X.G. Chen, X.B. Ma, J.Z. Han, Y.C. Yang, S. Guo, A.R. Yan, Q.Z. Huang, M.M. Wu, D.F. Chen, Anisotropic nanocrystalline MnBi with high coercivity at high temperature, Appl. Phys. Lett., 99 (2011) 082505.

[5] J.B. Yang, K. Kamaraju, W.B. Yelon, W.J. James, Q. Cai, A. Bollero, Magnetic properties of the MnBi intermetallic compound, Appl. Phys. Lett., 79 (2001) 1846-1848.

[6] N.V. Rama Rao, A.M. Gabay, G.C. Hadjipanayis, Anisotropic fully dense MnBi permanent magnet with high energy product and high coercivity at elevated temperatures, J. Phys. D: Appl. Phys., 46 (2013) 062001.

[7] Y.B. Yang, X.G. Chen, R. Wu, J.Z. Wei, X.B. Ma, J.Z. Han, H.L. Du, S.Q. Liu, C.S. Wang, Y.C. Yang, Y. Zhang, J.B. Yang, Preparation and magnetic properties of MnBi, J. Appl. Phys., 111 (2012) 07E312. [8] C. Guillaud, Polymorphism of the compound MnBi at the temperatures of disappearance and reappearance of the spontaneous magnetization, J. Phys. Radium, 12 (1951) 143-143.

[9] Y.L. Ma, X.B. Liu, K. Gandha, N.V. Vuong, Y.B. Yang, J.B. Yang, N. Poudyal, J. Cui, J.P. Liu, Preparation and magnetic properties of $\mathrm{MnBi}$-based hard/soft composite magnets, J. Appl. Phys. , 115 (2014) 17 A755.

[10] Y.Q. Li, M. Yue, T. Wang, Q. Wu, D.T. Zhang, Y. Gao, Investigation of magnetic properties of MnBi/Co and $\mathrm{MnBi} / \mathrm{Fe} 65 \mathrm{Co} 35$ nanocomposite permanent magnets by micro-magnetic simulation, Journal of Magnetism and Magnetic Materials, 393 (2015) 484-489. 


\section{Wei et al. on "Effect of ball milling and heat treatment process on MnBi powders magnetic properties JALCOM-D-15-09718"}

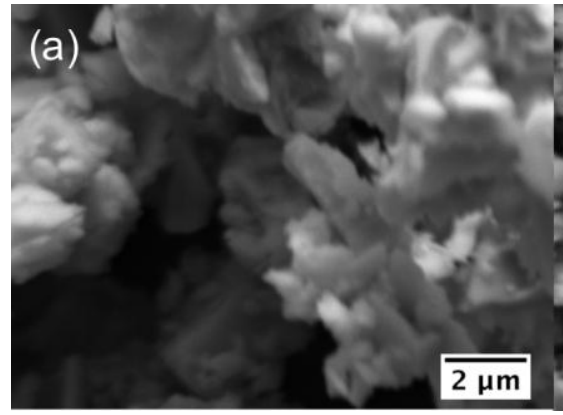
Magn., 49 (2013) 3387-3390. 1843. 374-381.

[11] J. Cui, J.P. Choi, G. Li, E. Polikarpov, J. Darsell, M.J. Kramer, N.A. Zarkevich, L.L. Wang, D.D. Johnson, M. Marinescu, Q.Z. Huang, H. Wu, N.V. Vuong, J.P. Liu, Development of MnBi permanent magnet: Neutron diffraction of MnBi powder, J. Appl. Phys., 115 (2014) $17 A 743$.

[12] P.K. Nguyen, S. Jin, A.E. Berkowitz, Unexpected Magnetic Domain Behavior in LTP-MnBi, IEEE Trans.

[13] M. Kishimoto, K. Wakai, Single-Domain Properties in MnBi Particles, Japan. J. Appl. Phys., 15 (1976)

[14] N.V. Rama Rao, A.M. Gabay, W.F. Li, G.C. Hadjipanayis, Nanostructured bulk MnBi magnets fabricated by hot compaction of cryomilled powders, J. Phys. D: Appl. Phys., 46 (2013) 265001.

[15] F. Yin, N. Gu, T. Shigematsu, N. Nakanishi, Sintering formation of low temperature phase MnBi and its disordering in mechanical milling, J . Mater. Sci. Technol., 12 (1996) 335-341.

[16] N.V. Rama Rao, A.M. Gabay, X. Hu, G.C. Hadjipanayis, Fabrication of anisotropic MnBi nanoparticles by mechanochemical process, J. Alloys Comp., 586 (2014) 349-352.

[17] M.P. Rowe, D. Herrera, New chemical synthesis of discrete MnBi hard magnetic nanoparticles, in: 247th ACS National Meeting \& Exposition, American Chemical Society, Dallas, TX, 2014, pp. INOR-144. [18] J. Cui, J.-P. Choi, E. Polikarpov, M.E. Bowden, W. Xie, G. Li, Z. Nie, N. Zarkevich, M.J. Kramer, D. Johnson, Effect of composition and heat treatment on MnBi magnetic materials, Acta Mater., 79 (2014)

228 Fig. 1 SEM morphology of ball milled powders for (a) $3 \mathrm{~h}$, (b) $8 \mathrm{~h}$ and (c) $24 \mathrm{~h}$

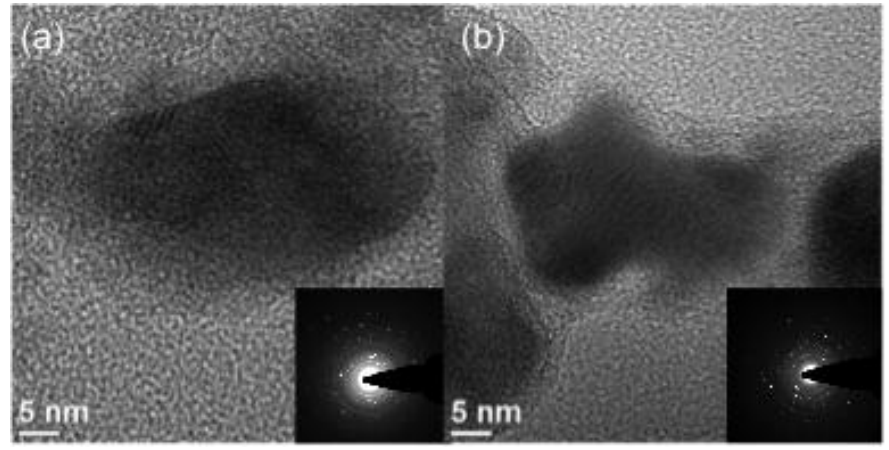

Fig. 2 TEM microscopy of the $\mathrm{Mn}_{55} \mathrm{Bi}_{45}$ powders ball milled for (a) 3 hours, (b) 24 hours 


\section{Wei et al. on "Effect of ball milling and heat treatment process on MnBi powders magnetic properties JALCOM-D-15-09718"}

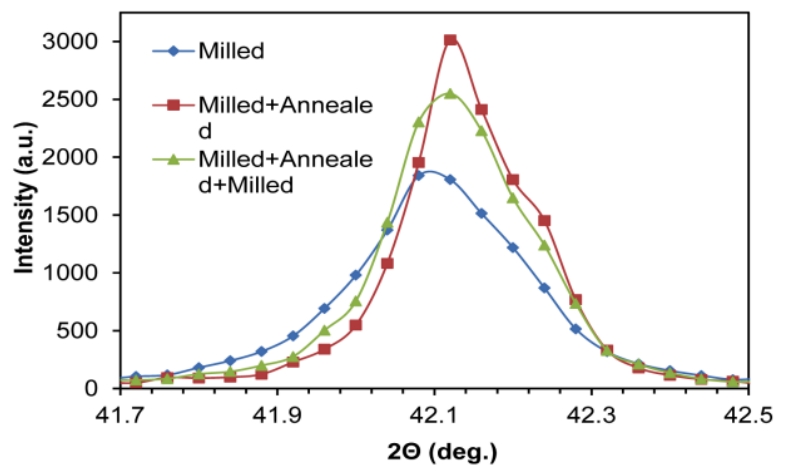

232 Fig. $3 \mathrm{X}$-Ray diffraction patterns of $\mathrm{Mn}_{55} \mathrm{Bi}_{45}$ powders ball milled for eight hours; milled for 233 eight hours and then heat-treated; and milled for eight hours, heat treated, and ball milled for 234 extra half an hour

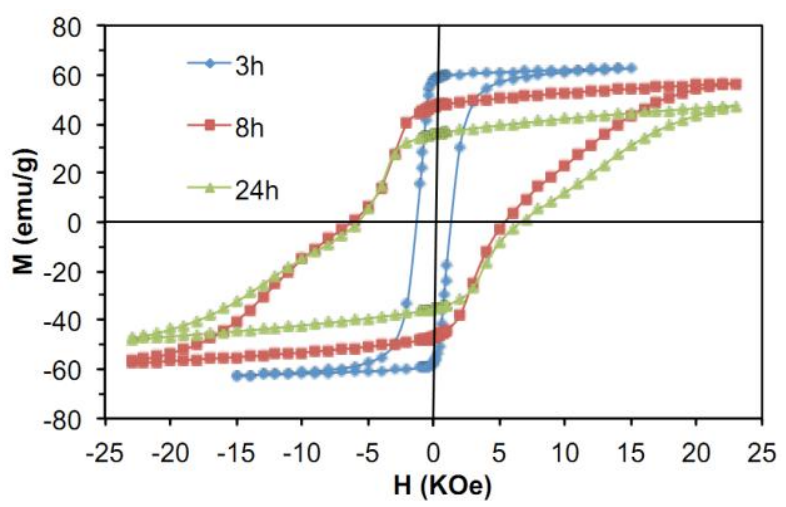

237 Fig. $4 \mathrm{M}-\mathrm{H}$ curves for $\mathrm{Mn}_{55} \mathrm{Bi}_{45}$ for varied ball milling durations

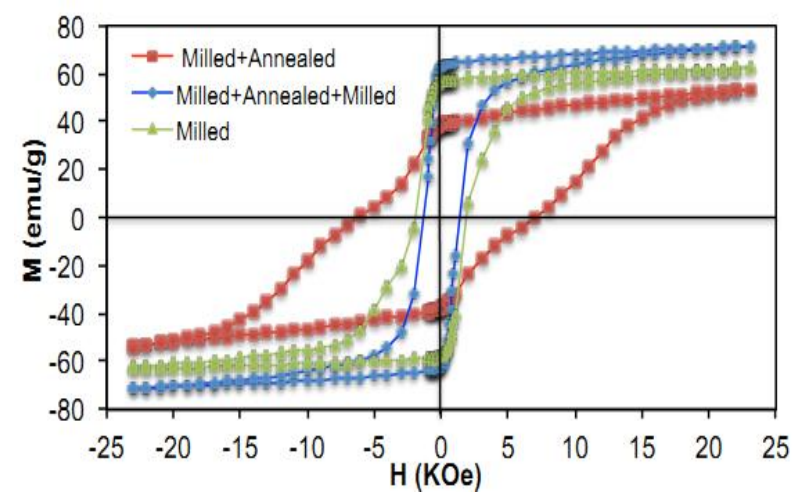




\section{Wei et al. on "Effect of ball milling and heat treatment process on MnBi powders magnetic properties JALCOM-D-15-09718"}

Fig. $5 \mathrm{M}-\mathrm{H}$ curves of $\mathrm{Mn}_{55} \mathrm{Bi}_{45}$ powders ball milled for eight hours; milled for eight hours and

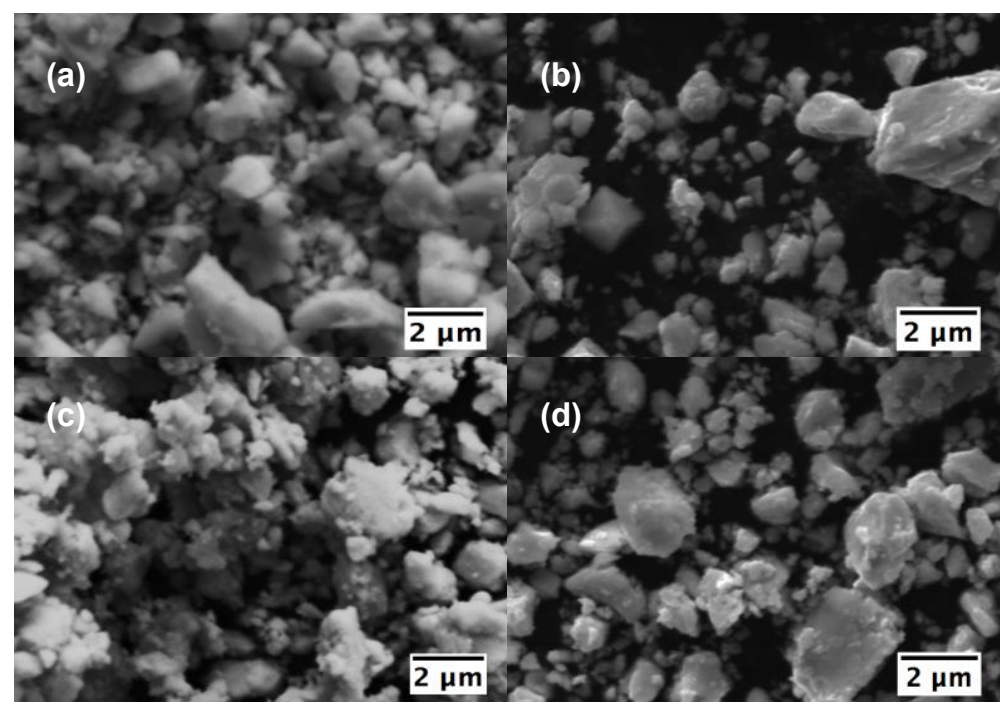

242 Fig. 6. The comparison of morphology of ball milled MnBi powders at (a) room temperature for

$2438 \mathrm{~h}$, (b) cryogenic environment for $8 \mathrm{~h}$, (c) room temperature for $24 \mathrm{~h}$, (d) cryogenic environment 244 for $24 \mathrm{~h}$

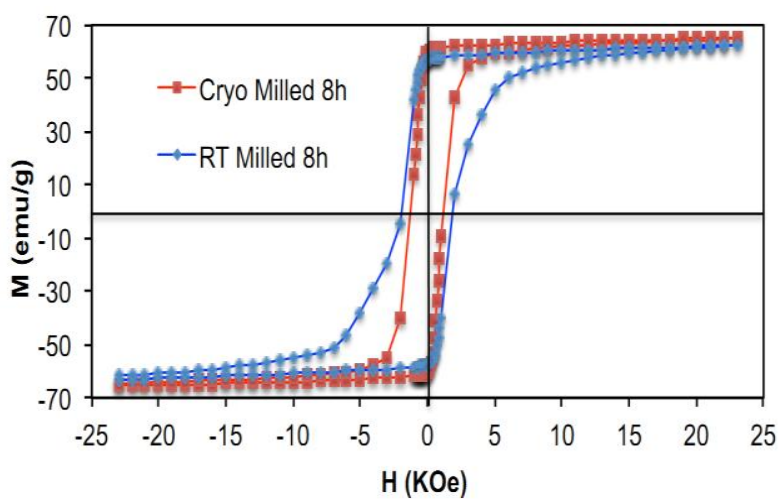

247 Fig. 7. M-H curves for 8 hours ball milled $\mathrm{Mn}_{55} \mathrm{Bi}_{45}$ at room temperature and cryogenic 248 environment 


\section{Wei et al. on "Effect of ball milling and heat treatment process on MnBi powders magnetic properties JALCOM-D-15-09718"}

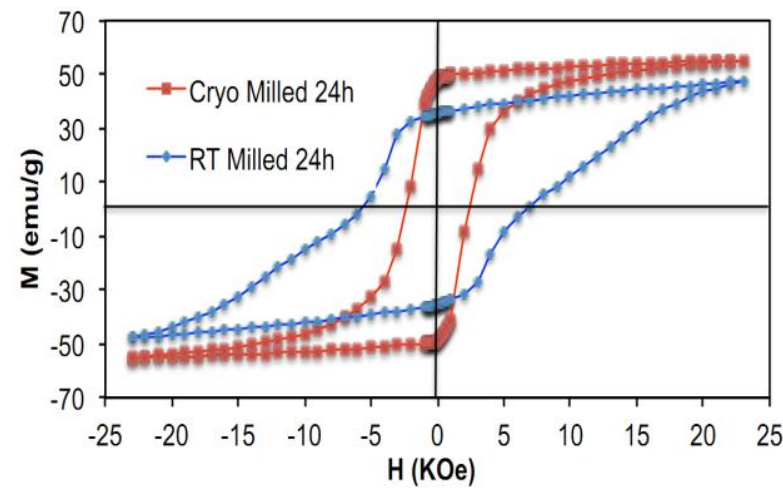

Fig. 8. M-H curves for 24 hours ball milled $\mathrm{Mn}_{55} \mathrm{Bi}_{45}$ at room temperature and cryogenic environment

252 Table $1 \mathrm{X}$-Ray phase composition analysis of MnBi powders ball milled at room temperature for eight hours; milled for eight hours and then heat-treated for twenty-four hours; and milled for eight hours, heat treated, and ball milled for extra half an hour. Uncertainties in the last significant figures are shown in parentheses.

\begin{tabular}{|c|c|c|c|c|c|c|c|c|c|}
\hline Sample & & & MnBi & & $\mathbf{B i}$ & & Mn & & MnO \\
\hline & Wt \% & a $(\stackrel{\AA}{)})$ & c $(\stackrel{\circ}{)})$ & $\begin{array}{c}\text { Crystallite size } \\
\text { (nm) }\end{array}$ & Wt \% & $\begin{array}{c}\text { Crystallite size } \\
(\mathbf{n m})\end{array}$ & Wt \% & $\begin{array}{c}\text { Crystallite size } \\
(\mathbf{n m})\end{array}$ & Wt \% \\
\hline Feedstock & $86.1(6) \%$ & $4.2867(1)$ & $6.1181(1)$ & $215(20)$ & $5.3(3) \%$ & & $2.4(3) \%$ & & $6.3(4) \%$ \\
\hline Milled & $77.0(5) \%$ & $4.2876(1)$ & $6.1178(2)$ & $52(1)$ & $13.7(2) \%$ & $39(1)$ & $5.0(4) \%$ & 49(9) & $4.3(4) \%$ \\
\hline $\begin{array}{c}\text { Milled } \\
\text { +annealed }\end{array}$ & $85.9(4) \%$ & $4.2866(1)$ & $6.1176(1)$ & $115(3)$ & $5.2(2) \%$ & $43(3)$ & $5.5(3) \%$ & $103(20)$ & $3.4(3) \%$ \\
\hline $\begin{array}{c}\text { Milled } \\
\text { +annealed } \\
\text { +milled }\end{array}$ & $81.0(4) \%$ & $4.2867(1)$ & $6.1177(1)$ & $85(2)$ & $10.2(2) \%$ & $39(1)$ & $4.5(3) \%$ & $89(18)$ & $4.3(3) \%$ \\
\hline
\end{tabular}




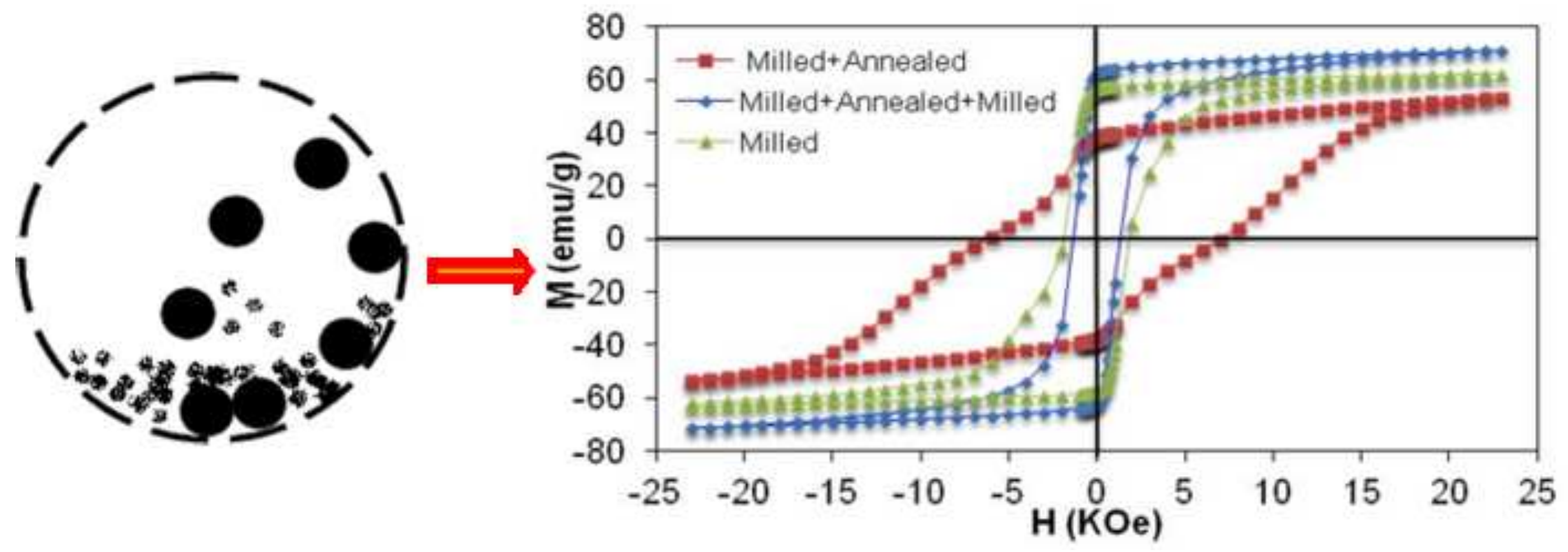

